\title{
Comercialização de plantas medicinais no contexto da cadeia produtiva em Minas Gerais
}

SOUZA, M.R.M. ${ }^{1 *}$; PEREIRA, R.G.F. ${ }^{2}$; FONSECA, M.C.M. ${ }^{1}$

${ }^{1}$ EPAMIG - Unidade Regional da Zona da Mata, Vila Gianetti, 46, Campus da UFV, CEP: 36570-000, Viçosa-

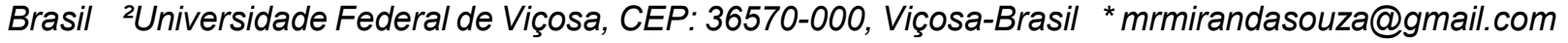

RESUMO: A Política Nacional de Práticas Integrativas e Complementares no Sistema Único de Saúde (SUS) contribuiu para o incremento do uso e conhecimento das plantas medicinais brasileiras, incentivando e respaldando o cultivo por agricultores familiares e a inserção no mercado. O Programa de Desenvolvimento de Plantas Medicinais prioriza ações em toda a cadeia produtiva do cultivo à distribuição. $O$ conhecimento da demanda e dos padrões de qualidade adequados permite que distribuidores e consumidores possam tomar decisões sobre a aquisição e oferta dos produtos medicinais. Entretanto, há carência de informações e tecnologias na cadeia produtiva, o que desfavorece o seu pleno desenvolvimento, condizente com o potencial de geração de renda e promoção da saúde. O objetivo deste trabalho foi identificar e analisar o padrão atual de obtenção e comercialização de plantas medicinais no Mercado Central de Belo Horizonte. Foram realizadas entrevistas semi-estruturadas com amostra representativa de 9 comerciantes das 23 lojas de plantas medicinais do Mercado Central, de acordo com o critério de acessibilidade descrito por Berquó. Os resultados demonstraram a precariedade desse setor quanto à obtenção das plantas medicinais, principalmente pela falta de conhecimento e informações na aquisição e venda dos produtos pelos consumidores e vendedores, respectivamente.

Palavras-chave: comercialização, plantas medicinais, cadeia produtiva, mercado

ABSTRACT: Commercialization of medicinal plants in the context of the productive chain in Minas Gerais. The National Politic Integrative and complementary Practices in Health System (SUS) contributed to the increased use and knowledge of Brazilian medicinal plants, which encourages and supports the growing of family farmers and their integration in efficient markets. The Program for the Development of Medicinal Plants prioritizes actions in the entire production chain from cultivation to distribution. Knowledge of demand and adequate quality standards allows distributors and consumers to make decisions on the acquisition and supply of medicinal products. However, there is a lack of information and technologies in this supply chain, which discourages its full development, consistent with the potential for income generation and health promotion presents. The objective of this study was to identify and analyze current patterns of production and marketing of medicinal plants in the Central Market of Belo Horizonte. We conducted semistructured interviews with nine of the 23 stores trades of medicinal plants from the Central Market. Results showed the precarious nature of this sector in obtaining medicinal plants, mainly by lack of knowledge and information in the purchase and sale of products by consumers and sellers, respectively.

Key words: comercialization, medicinal plants, productive chain, market

\section{INTRODUÇÃO}

Com a adoção da Política Nacional de Práticas Integrativas e Complementares no Sistema Único de Saúde (SUS), está reaberto o portal de acesso ao conhecimento das plantas medicinais brasileiras. Por meio do PPPM (Programa de Pesquisas de Plantas Medicinais), tornou-se possível associar o saber popular ao saber técnico-científico, com o empenho de pesquisadores brasileiros e apoio dos Ministérios da Ciência e Tecnologia, da Saúde, do Meio Ambiente e da Agricultura, integrados na implantação de um Programa de Desenvolvimento de Plantas Medicinais, visando priorizar ações desde o

Recebido para publicação: setembro de 2011

Aceito para publicação: março de 2012

Rev. Bras. PI. Med., Botucatu, v.14, n.esp., p.242-245, 2012. 
cultivo até a comercialização e distribuição de plantas medicinais (Brasil, 2006a).

Essa política contribuiu para o incremento do uso de plantas medicinais, o que pode incentivar e respaldar o cultivo por agricultores familiares, permitindo sua inserção na cadeia produtiva, de forma eficiente e adequada, e o acesso aos mercados tradicionais e, ou potenciais. Conhecendo a demanda e os padrões de qualidade previamente estabelecidos, o produtor pode tomar decisões sobre o que, como, quanto e quando produzir. Entretanto, ainda há carência de informações e tecnologias nessa cadeia, o que desfavorece a obtenção de um produto final nestes padrões.

O Decreto no 5813, publicado em 2006, aprova a Política Nacional de Plantas Medicinais e Fitoterápicos (PNPMF) (Brasil, 2006a) sendo um dos principais instrumentos norteadores para 0 desenvolvimento de ações e programas de plantas medicinais e fitoterapia na saúde pública. Este Decreto traz diretrizes para desenvolvimento da cadeia produtiva de plantas medicinais e fitoterápicos (Brasil, 2006a). Já a Política Nacional de Práticas Integrativas e Complementares (PNPIC) no SUS, contempla diretrizes, ações e responsabilidades dos entes federais, estaduais e municipais para inserção de serviços na rede pública, dentre outras práticas, as plantas medicinais e a fitoterapia (Brasil, 2006b; Brasil, 2008). As ações decorrentes dessa Política, manifestadas em um programa, são imprescindíveis para melhorar: o acesso da população às plantas medicinais e fitoterápicos; a inclusão social e regional; o desenvolvimento agrícola, industrial e tecnológico; a promoção da segurança alimentar e nutricional; o uso sustentável da biodiversidade brasileira e a valorização/preservação do conhecimento tradicional das comunidades e povos tradicionais (Brasil, 2009).

O programa Componente Verde da Rede Farmácia de Minas lançado pela Secretaria Estadual da Saúde em Minas Gerais em março de 2010, também é um canal de acesso às plantas medicinais através do Sistema Único de Saúde (SUS). Para iniciar o trabalho de inserção da fitoterapia no SUS do estado, o "Componente Verde" selecionou 16 espécies de plantas medicinais, a saber: Allium sativum L. (alho), Calendula officinalis L. (calêndula), Cordia verbenacea DC. (erva-baleeira) Cynara scolymus L. (alcachofra), Lippia sidoides Cham. (alecrim-pimenta), Maytenus ilicifolia (Schrad.) Planch. (espinheira-santa), Melissa officinalis L. (melissa), Mentha x piperita L. (hortelã-pimenta), Mentha villosa Huds (hortelã-rasteira), Mikania laevigata (guaco), Ocimum gratissimum L. (alfavaca), Passiflora alata Curtis (maracujá-doce), Passiflora edulis Sims (maracujá-azedo), Passiflora incarnata L. (maracujásilvestre), Plantago major L. (tanchagem) e
Stryphnodendron adstringens (Mart.) Coville (barbatimão). As espécies listadas acima foram préselecionadas por meio de validação científica. Este programa enfatiza a necessidade de controle de qualidade de maneira equânime entre os elos da cadeia de plantas medicinais, da produção até o consumidor final (Sant'Ana et al., 2009).

Apesar da demanda por espécies de uso medicinal, há carência de informações, principalmente por parte do produtor, o que pode ser observado também nos demais setores, basicamente indústria, comércio e consumidores. O Brasil, ao contrário do que ocorre nos países europeus, asiáticos e nos Estados Unidos, praticamente não dispõe de estatísticas que expliquem o mercado, o consumo e os costumes de uso de plantas medicinais, apesar de existir tradição no uso. A falta de informação contribui para a produção e obtenção de um produto vegetal de má qualidade (Veiga Junior, 2008). Como em qualquer sistema de produção agroindustrial, um ponto fundamental para o sucesso do empreendimento rural familiar é o acesso a mercados potenciais, o qual ocorre por meio do conhecimento da demanda e possibilita a definição de padrões de qualidade, quantidades dos produtos e planejamento da produção.

A origem da matéria-prima vegetal utilizada é de grande relevância para a garantia da qualidade e segurança das espécies medicinais. Isto depende, em primeiro lugar, das matrizes, as quais devem ser adquiridas de instituições idôneas e identificadas botânicamente, impondo menor risco ao cultivo e à posterior utilização terapêuica.

A exploração de plantas medicinais na flora nativa por meio de extração direta nos ecossistemas tropicais (extrativismo) é um grave problema, e tem levado a drásticas reduções das populações naturais destas espécies, seja pelo processo predatório da exploração, seja pelo desconhecimento de mecanismos de perpetuação (Reis \& Mariot, 2002). Assim, a domesticação e o cultivo se tornam a melhor opção para obtenção da matéria-prima vegetal em bases sustentáveis.

Considerando-se o valor das plantas medicinais não apenas como recurso terapêutico, mas também como fonte de renda para a agricultura familiar, torna-se importante estabelecer linhas de ação voltadas ao desenvolvimento de técnicas de manejo sustentável ou cultivo, visando à utilização destas espécies, aliada à manutenção do equilíbrio dos ecossistemas tropicais (Reis, 1996; Sheldon et al., 1997). Deve ser considerado ainda que a produção agrícola está sujeita a restrições devido às variações das condições edafoclimáticas e que o teor de princípios ativos, e portanto, as propriedades terapêuticas e condimentares, podem ser alteradas devido a diversos fatores como o método de secagem e beneficiamento pós-colheita, época do ano e local

Rev. Bras. Pl. Med., Botucatu, v.14, n.esp., p.242-245, 2012. 
de cultivo (Court et al., 1993; Fonseca et al., 2006), refletindo o efeito do meio ambiente sobre a produção dos princípios fármaco-ativos (Corrêa Júnior et al., 1991).

Quanto à comercialização, Lourenzani et al. (2004) argumentam que o mercado de plantas medicinais poderia ser estruturado de forma mais eficiente observando-se a existência de três diferentes canais de comercialização: a) informal; b) farmácias de manipulação e c) indústrias. Esses canais devem satisfazer a demanda por meio do fornecimento de mercadorias e serviços no lugar, quantidade, qualidade e preços adequados.

O objetivo desse trabalho foi identificar o padrão atual de obtenção e comercialização das plantas medicinais em um setor específico, o Mercado Central de Belo Horizonte, com o intuito de fornecer subsídios para estabelecer um possível canal de comercialização e apresentar informações para o setor produtivo de Minas Gerais.

\section{MATERIAL E MÉTODO}

Realizaram-se entrevistas semi-estruturadas, as quais abrem espaço para a complementação das questões do roteiro básico, de acordo com as circunstâncias no momento da entrevista, o que permite emergir informações de forma livre, no âmbito dos objetivos (Manzini, 1990; Triviños, 1997). O roteiro foi elaborado com base em questões relacionadas às condições de oferta e demanda das plantas medicinais pelos comerciantes e consumidores, informações relacionadas à compra e venda dos produtos medicinais, padrões de preço, quantidade, qualidade e fatores que influenciam a disponibilidade dos produtos no mercado.

Foram realizadas entrevistas com 9 comerciantes de diferentes firmas nos 23 pontos comerciais de plantas medicinais existentes no Mercado Central de Belo Horizonte (MG), de acordo com o critério de acessibilidade (Berquó et al., 2006), para evidenciar a dinâmica e as condições de oferta e demanda relacionadas à comercialização das principais espécies medicinais. Os entrevistados comercializam principalmente ervas in natura, além de alguns produtos em forma de pomadas, e essências, dentre outros.

Para realização desta pesquisa, foram selecionadas algumas espécies registradas pela ANVISA e que fazem parte do Programa Estadual Componente Verde: alcachofra (Cynara scolymus.), alecrim (Rosmarinus officinalis), alfavaca (Ocimum gratissimum), alho (Allium sativum), barbatimão (Stryphnodendron adstringens), calêndula (Calendula officinalis), erva-baleeira (Cordia verbenacea), espinheira santa (ilicifolia), guaco (Mikania laevigata), hortelã (Mentha x villosa), maracujá (Passiflora edulis), melissa (Melissa officinalis) e tanchagem (Plantago major).

\section{RESULTADO E DISCUSSÃO}

Os resultados demonstraram a precariedade do setor quanto à obtenção das plantas medicinais, principalmente pela falta de conhecimento e informações na aquisição e venda dos produtos pelos consumidores e vendedores, respectivamente. A mídia é a referência principal de informação para compradores de ervas medicinais e derivados, mas nem sempre valida o uso tradicional, ou é coerente na divulgação de resultados científicos sobre os mesmos.

Das 16 espécies selecionadas do Programa Componente Verde, todas estavam disponíveis para venda nos estabelecimentos; apenas dois estabelecimentos não dispunham de alho para venda. Quanto à época de maior procura, percebeu-se que a mídia televisiva e impressa, revistas de uma maneira geral, contribuem para o aumento da procura, havendo resposta direta quando há lançamento de alguma matéria jornalística. Entretanto as informações chegam de forma incompleta, resultando em equívocos quanto à identificação correta e consequentemente quanto ao uso medicinal.

Alguns entrevistados relataram que as informações dos compradores são vagas, pois não apresentam prescrição de algum especialista e tomam como base o que a mídia apresenta.

As informações fornecidas pelos balconistas geralmente tiveram como base a própria experiência, os quais, ao serem questionados, não demonstraram apresentar nenhum tipo de capacitação para realizar esta tarefa.

As espécies mais procuradas foram alecrim (7), hortelã (5), guaco (5), sendo este mais procurado em época de inverno, barbatimão (5), tanchagem (5), espinheira santa (4) e erva-baleeira (4). A aquisição destas espécies foi considerada, de maneira geral pelos comerciantes, dificultada pela necessidade de buscar mercados externos a Minas Gerais, e a dependência de empresas de São Paulo. Apesar de estarem cientes da existência de produtores rurais na região que poderiam fornecer as ervas medicinais, manifestaram não ter informações que pudessem permitir o acesso a eles.

Um dos comerciantes citou como outro problema na aquisição das plantas a situação da certificação da qualidade, que no geral foi considerada baixa. Foi relatado que o fornecimento das plantas é feito por empresas de São Paulo na maioria (8); apenas um comerciante citou fornecimento de empresa do Rio Grande do Sul. Outros fornecimentos ocorrem por intermédio de raizeiros da região de Belo Horizonte (4) ou provenientes de cultivo próprio (1). Quanto à qualidade, 2 comerciantes disseram saber informar com certeza e apenas 2 afirmaram que as empresas fornecedoras enviam análise das plantas. A compra dos produtos nas lojas não especifica um público

Rev. Bras. PI. Med., Botucatu, v.14, n.esp., p.242-245, 2012. 
preferencial. A venda é constante (2), na virada de ano ou em datas especiais (2) época de inverno (3), não há época específica (1) e por meio da mídia (1). É interessante destacar que a mídia é citada como o principal veículo de informação sobre as plantas medicinais, sendo que a resposta à procura é imediata quando alguma propaganda é divulgada. No geral os compradores (consumidores) não mostram conhecimento sobre as plantas que adquirem. A forma mais comum do produto vegetal ofertado são ervas secas, em pacotes de 20-40 g e vendidos na faixa de $R \$ 1,50$ a $R \$ 2,00$.

Os resultados demonstraram a precariedade da cadeia produtiva no que diz respeito à obtenção das plantas medicinais, principalmente pela falta de conhecimento, capacitação, informações na aquisição e venda dos produtos pelos consumidores e vendedores, respectivamente, e de interação entre os canais de comercialização. Destacou-se a mídia como fonte importante de informações, estimulando ou desestimulando a compra de produtos à base de plantas medicinais. No entanto, este veículo de comunicação nem sempre está relacionado à validação do uso tradicional ou à divulgação idônea de resultados científicos sobre o uso de espécies medicinais.

Acredita-se que o aumento do consumo das plantas medicinais e as políticas e programas governamentais devam ser vistos como oportunidades para alternativas de renda na produção familiar, e portanto devem ser melhor divulgados, diante da falta de conhecimentos e informações constatadas na aquisição e venda dos produtos.

A mídia como canal de comunicação não deve servir de base de validação do uso tradicional ou de divulgação de resultados científicos sobre o uso de plantas medicinais. Há carência de comunicação efetiva entre os canais de distribuição e produção; diante desse quadro é necessário construir uma rede eficiente de divulgação das plantas medicinais, o que poderia ser estabelecido por políticas de comunicação integradas ao Programa de desenvolvimento de plantas medicinais.

\section{AGRADECIMENTO}

À Fundação de Amparo e Pesquisa do Estado de Minas Gerais pelo apoio financeiro.

\section{REFERÊNCIA}

BERQUÓ, E.S.; SOUZA, J.M.P.; GOTLIEB, S.L.D. Bioestatística. São Paulo: EPU. 2.ed. 2006. 350p.

BRASIL. Ministério da Saúde. Secretaria de Ciência, Tecnologia e Insumos Estratégicos. Departamento de Assistência Farmacêutica. A fitoterapia no SUS e o Programa de Pesquisa de Plantas Medicinais da Central de Medicamentos. Brasília: Ministério da Saúde, 2006a. 148p. (Série B. Textos Básicos de Saúde).

BRASIL, Ministério da Saúde. Portaria no 971 de 03 de Maio de 2006b. Aprova a Política Nacional de Práticas Integrativas e Complementares (PNPIC) no SUS. Disponível em: <http://189.28.128.100/dab/docs/legislacao/ portaria971_03_05_06.pdf>. Acesso em: out. 2010.

BRASIL. Portaria Interministerial no 2.960, de 09 de dezembro de 2008. Aprova o Programa Nacional de Plantas Medicinais e Fitoterápicos e cria o Comitê Nacional de Plantas Medicinais e Fitoterápicos. Diário Oficial [da] República Federativa do Brasil, Brasília, DF, 10 dez. 2008a. Seção 1. p.56.

BRASIL. Ministério da Saúde, Secretaria de Ciência e Tecnologia e Insumos Estratégicos, Relação Nacional de Medicamentos Essenciais - Rename, 6.ed. Brasília: Ministério da Saúde, 2009. (Série B. Textos Básicos de Saúde).

CORREA JÚNIOR, C.; MING, L.C.; SCHEFFER, M.C. Cultivo de plantas medicinais, condimentares e aromáticas. Curitiba: EMATER-PR, 1991, 151p.

COURT, W.A.; ROY, R.C.; POCS, R. Effect of harvest date on the yield and quality of the essential oil of peppermint. Canadian Journal Plant Science, v.73, p.815-24, 1993. FONSECA, M.C.M.; CASALI, V.W.D.; STROZI, R.M.M. Anatomia dos órgãos vegetativos e histolocalização de compostos fenólicos e lipídicos em Porophyllum ruderale (Asteraceae). Revista Planta Daninha, v.24, n.4, p.707-13, 2006.

LOURENZANI,A.E.B.S.; LOURENZANI, W.L.L.; BATALHA, M.O. Barreiras e oportunidades na comercialização de plantas medicinais provenientes da agricultura familiar. Informações Econômicas, v.34, n.3, p.15-25, 2004.

MANZINI, E.J. Considerações sobre a elaboração de roteiro para entrevista semi-estruturada. In: MARQUEZINE, M.C.; ALMEIDA, M. A.; OMOTE, S. (Orgs.). Colóquios sobre Pesquisa em Educação Especial. Londrina: Eduel, 2003. p.11-25.

TRIVIÑOS, A.N.S. Introdução à pesquisa em ciências sociais: a pesquisa qualitativa em educação. São Paulo: Atlas, 1997. 176p.

REIS, M.S. Manejo sustentado de plantas medicinais em ecossistemas tropicais. In: DI STASI, L.C. (Org.). Plantas medicinais: arte e ciência - Um guia de estudo interdisciplinar. São Paulo: USP, 1996. p.198-214.

REIS , M.S.; MARIOT, A. Diversidade natural e aspectos agronômicos de plantas medicinais. In: SIMÕES, C.M. et al. (Orgs.). Farmacognosia: da planta ao medicamento. Porto Alegre/Florianópolis: UFRGS/UFSC, 2002. p.41-62. SANT'ANA, J.M.B. et al. Essencialidade e assistência farmacêutica: considerações sobre o acesso a medicamentos mediante ações judiciais no Brasil. Revista Panamericana de Salud Publica, v.29, n.2, p.138-44, 2011.

SHELDON, J.W.; BALICK, M.J.; LAIRD, S.A. Medicinal Plants: can utilization and conservation coexist? New York: New York Botanical Garden, 1997. 104p.

VEIGA JUNIOR, V.F. Estudo do consumo de plantas medicinais na Região Centro-Norte do Estado do Rio de Janeiro: aceitação pelos profissionais de saúde e modo de uso pela população. Revista Brasileira de Farmacognosia, v.18, n.2, p.308-13, 2008. 